\title{
Xanthogranulomatous Pyelonephritis: a case report
}

\author{
GBL Samarasekera ${ }^{1}$,Satish Goonesinghe ${ }^{2}$, JDVC Lekamwasam ${ }^{3}$ \\ ${ }^{1}$ Registrar in Medicine, ${ }^{2}$ Consultant Genito-Urinary Surgeon, ${ }^{3}$ Consultant Physician, Teaching Hospital, Galle.
}

e-mail address of the corresponding author,Dr.G.B.L. Samarasekera: bodhika@yahoo.com

\section{Introduction}

Xanthogranulomatous pyelonephritis is a rare debilitating illness which was first described in 1916 by Schlangenhaufer. It ultimately results in diffuse or focal renal destruction and share many features of renal cell carcinoma. Histologically, disease is characterized by lipid laden foamy macrophages

The disease is more common in middle aged females. Most cases are unilateral, and often associated with urinary tract obstructions (Staghorn calculi), urinary tract infections and diabetes. It is commonly associated with Proteus mirabilis and Escherichia coli infections.

\section{Case report}

A 72 year-old housewife was admitted with high grade fever, with chills and rigors. She also had several episodes of vomiting including one episodes of haematemesis.

At the time of the admission she did not complain of dysuria, increased frequency, or haematuria. But she had been taking treatment for recurrent urinary tract infections in the recent past.

She experienced dull pain in her left loin. This was intermittently present during past eight months. This illness was associated with loss of weight and loss of appetite. She was a hypertensive and on regular treatment.

On examination, she was in pain and was also febrile and pale. Her blood pressure was $110 / 70 \mathrm{mmHg}$. Abdominal examination revealed left side loin tenderness.

Initial haematological investigations revealed WBC / DC 18,800 with $92 \%$ neutrophils. Her ESR was 150 $\mathrm{mm}$ in first hour and it was repeatedly high. Her urinalysis showed moderately field full of pus cells. Her urine culture was positive for E. coli and the blood urea was initially high and later became normal.

Her ultrasound abdomen showed an enlarged left kidney with multiple calculi and dilatation of the pelvicalyceal system. There were hypoechoic areas seen inside the dilated system. Features were suggestive of xanthogranulomatous pyelonephritis

The patient was treated with IV co-amoxiclav for two weeks. Ultrasound scan was repeated and the appearances were same as in the previous scan.

$\mathrm{CT}$ abdomen revealed ureteric stone measuring 1.5 $\mathrm{cm} 1 \mathrm{~cm}$ in the left renal pelvis. The left kidney was enlarged with multiple lobulated appearance, thickened walls and septations. It showed poor contrast excretion comparatively to right. The fascia around left kidney was thickened and this shows probability of xanthogranulomatous with its bear's paw appearance.

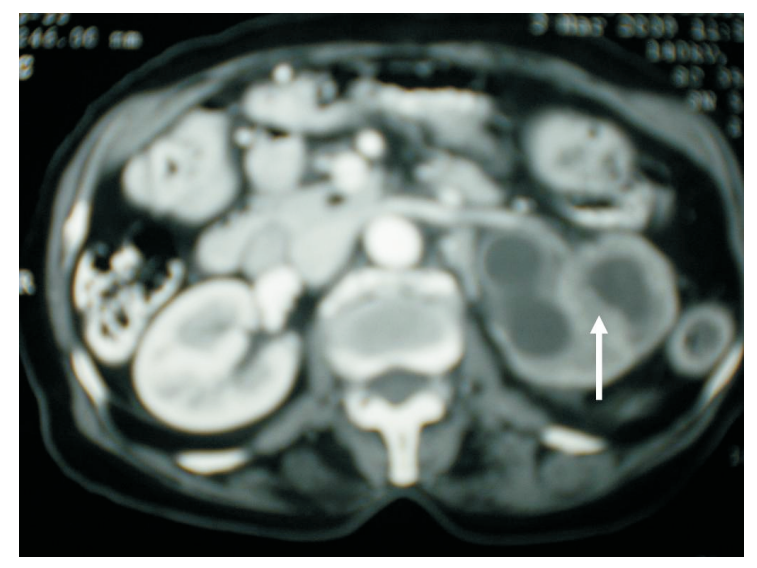

Figure 1 - Contrast enhanced CT scan of abdomen showing bear's paw appearance of left kidney due to Xanthogranulomatous pyelonephritis 


\section{Discussion}

Xanthogranulomatous pyelonephritis usually presents with anorexia, fever, chill, weight loss and flank pain. Urine contains both leucocytes and organisms. Culture may be positive for Proteus or $E$. coli. However in one third of patients the culture is negative. Our patient's urine culture was positive for E. coli. This can be precipitated by urinary tract obstruction as in our patient.

Neutrophil leucocytosis and anaemia are common findings as in our case. Liver function tests are abnormal in up to $50 \%$ of patients. Renal ultrasound usually reveals multiple hypoechoic masses, irregular thin parenchyma and dilated collecting system. CT scan is the most useful radiographic imaging technique. CT scan demonstrates a heterogenous nonenhancing mass on hydronephrotic non-functioning kidney with a central stone .This gives characteristic bear's paw appearance as in our patient.

Mercaptotriglycine (MAG 3) or technetium - 99m dimercapto succinic acid (TC-DMSA) scanning may be used to evaluate renal functions.

Medical therapy with antibiotics has only limited value, but antibiotics are prescribed as a temporizing measure prior to nephrectomy. Nephrectomy is the standard treatment for xanthogranulomatous pyelonephritis. Partial nephrectomy can be performed if the disease is limited to one area. The goal is to remove all granulomatous tissues. If this is not accomplished, the remaining infected tissues may lead to cutaneous fistula formation.
Laparoscopic nephrectomy is feasible in certain cases. Patients should be followed up since this can recur in other normal kidney. Annual imaging and aggressive treatment of all urinary tract infections are essential.

\section{References}

1. Ewing K Song, Mark Zwanger. Xanthogranulomatous Pyelonephritis. Journal of Emergency Medicine 2001; 21: 63-4.

2. R Loffroy, B Guiu J. Watfa F. Michel JP. Cercueil D Krause. Xanthogranulomatous in adults; clinical and Radiological finding in diffuse and focal forms. Clinical Radiology 2007; 62: 884-90.

3. Motaz Qadan, Michel Wines, David A. Folley. Xanthogranulomatous pyelonephritis no longer contraindication for Laparoscopic surgery. British Journal of Medical and Surgical Urology 2009; 2(1): 34-8.

4. John Chul Kim. US and CT findings of Xanthogranulomatous. Pyelonephritis Journal of Clinical Imaging 2001; 25: 118-21.

5. Chang I Ho, Yao Ko Wen, Mei Ling Chen. Xanthogranulomatous pyelonephritis successfully treated with antibiotics only: a case report. Journal of China Medical Association 2008; 71(12): 641-5. 